\title{
The Modelling of Pedestrian Vehicle Interaction for Post-Exiting Behaviour
}

\author{
Peter J Lawrence, Veronica Pellacini, Edwin R Galea \\ Fire Safety Engineering Group, University Of Greenwich, \\ Old Royal Naval College, London,UK \\ P.J.Lawrence@gre.ac.uk; V.Pellacini@gre.ac.uk; E.R.Galea@gre.ac.uk
}

\begin{abstract}
During a major evacuation of high capacity buildings, such as a tower block or transportation hub, the emergency services will need to consider the safety of the people within the vicinity of the emergency. However, in general, when assessing the safety of a building for evacuation only the behaviour within the building is considered. One method of assessing this is to utilise a computer based simulation tool. This research outlines a number of developments required to simulate the impact of traffic on the evacuation process in an urban environment in relation to post-exiting behaviour. It uses a unique data set and model capabilities for representing pedestrian-vehicle interaction post-evacuation, which also considers the impact of time pressures on decision making. In addition, a number of software developments and pedestrian behaviours are identified for bridging the behavioural gaps when interfacing an emergency pedestrian model with a traffic simulation.
\end{abstract}

Keywords: Pedestrian, Vehicle, Post-exiting behaviour, Road crossing, Time pressure

\section{Introduction}

The standard practice when assessing a building evacuation is that people are considered to have reached a place of safety once they have exited the building, which is typically used to calculate a required safe egress time [1]. However, in real emergencies, for example, the Holborn station fire and the Paddington station false alarm [2], the emergency services will need to also consider the safety of pedestrians within the vicinity of the facility [3]. In these cases, people will be evacuating into an urban environment where the road network and pedestrian space will impact people trying to clear the area or reach a place of safety, such as an assembly location.

The work presented here extends the building evacuation modelling domain to include the road network outside the facility being modelled as well as key behaviours in pedestrian-vehicle interaction. These developments are an attempt to model people's post-exiting behaviour, while in the vicinity of an emergency. The objective of this work is to answer the research question, what behaviours need to be implemented in a computer model to simulate people interacting with vehicles after exiting from a building in an emergency?

The developments include pedestrian behaviours such as selecting whether to use a pedestrian crossing, crossing behaviour within or outside (i.e. jaywalking) a designated crossing area, along with different strategies for how to cross, for example crossing each lane of traffic in stages. Furthermore, these behavioural developments investigate how time pressure may play a role in decision making associated with pedestrian road crossing, something which is not currently considered by other pedestrian evacuation models, which include vehicles [4]-[6]. In addition, the necessary behavioural developments to facilitate the coupling of buildingEXODUS [7][8] to a third-party vehicle evacuation model for the simulation of urban scale evacuations are discussed.

\section{Vehicle and Pedestrian Models}

There are a number of models which simulate pedestrian interaction with vehicles, such as LEGION [6], VISSIM [4] and MATSIM [5]. However, these models do not consider psychological factors such as the time pressure a person might feel during an actual emergency situation (i.e. a fire). Furthermore, they consider pedestrians crossing only at designated locations (pedestrian crossings) and do not at 
non-designated locations, as suggested by Wang [9] and rolling gap behaviours [10], which can be used if the pedestrian is considered a risk-taker [11][12][13].

Other research has been carried out looking at developing a framework for integrating fire, pedestrian and vehicle models for Wildland-Urban Interfaces [14]. While others have considered the vehicles as stationary obstacles for the evacuation [15]. The model presented here takes the recommendations identified by Schroeder [16] and Wang [9]. It then extends these ideas further with information from an online survey [17] to build a unique set of behaviours for representing how people might interact with vehicles in an emergency situation after evacuating from a building.

The cognitive behaviours for pedestrian road crossing included in this research cover the decision making process of whether to cross the road or not (gap acceptance model) and where to cross (at a pedestrian crossing or not). In addition, the methods employed to reach such decisions are considered. For further information on previous studies on pedestrian crossing behaviours related to cognitive aspects, see [18] and [19].

\section{Vehicle Modelling}

The main focus of the research developments within the evacuation model are on the pedestrian behaviours. Therefore, the primary purpose of the vehicle modelling is to provide interaction events for the pedestrians, so that pedestrian-vehicle interaction can take place. The vehicle modelling capability is limited to straight sections of the road network. The intention is to link these sections of the road network into a third-party vehicle model at a later date, when it is necessary to model the macro simulation of the road network. To allow for testing and development, without a third-party vehicle model, buildingEXODUS has been adapted to generate vehicles at specific locations in the model, at a user definable exponential rate [9] using a fuzzy logic car following model [20]. The road network can be imported from OpenStreetMap data [21], together with crossing information.

\section{Pedestrian and Vehicle Interaction}

The main simulation objectives of this research is to model people evacuating to a place of safety outside a building in an urban setting, where this place of safety is remote from the building itself. In these situations it is possible for people to come into contact with traffic and cross roads to leave the current area to reach the place of safety. For the crossing behaviour, a pedestrian crossing choice model has been developed which takes into account the pedestrian's distance from the pedestrian crossing, the type of crossing, time pressures, road type and traffic conditions and directions.

In the model, simulated people or agents, can select to cross a road at pedestrian crossing locations or at any point along the road. If an agent does not choose to use any of the available crossings, a probabilistic gap acceptance model is used which utilises the data from a number of previous studies [9][16][22], see Section 4.4. The gap acceptance model allows the pedestrian agent to choose to cross all lanes, when safe to do so, in one go, known as double gap [11] or one stage crossing [13]. Alternatively, if the agent is identified as a risk-taker, they may choose to cross lane by lane, rather than crossing all lanes in one go, sometimes waiting in the middle of the road, known as rolling gap [10] or risk-taker [11] crossing behaviour.

\subsection{Where to Cross}

Since the model is intended to be used to simulate people in emergency situations, a time pressure factor needs to be considered [23]. Furthermore, Chu et al [24] states a time pressure factor is important, because it has an influence on where and when people may cross a road. However, none of the models surveyed, as part of this research, were found to include such a feature. When examining other models there was lack of information regarding under what circumstances their data was collected, such as traffic conditions or there was vagueness regarding the travel distances involved. Therefore, the data needed for the crossing behaviour was collected using an online survey. The survey covered pedestrian crossing type, 
route choice, traffic conditions, and how this could be impacted if the person was in a hurry, see reference [17].

From the data collection, analysis probabilities were derived depending on the origin and destination position for the condition busy and light traffic and for each combination of rush and no rush.

The derivation of these curves will be discussed in a following article, however an example is shown in Figure 1 to indicate the data type employed and the method used. In Figure 1 the distance of the position of the agent from the pedestrian crossing is on the $\mathrm{x}$-axis while each line is for a different distance of the destination distance (bus stop) from the pedestrian crossing. For example, in Option 1, the probability to use the pedestrian crossing, starting at $40 \mathrm{~m}$ and arriving at $30 \mathrm{~m}$ (light grey line, Figure 1) in busy traffic rush is around $60 \%$. Similar probability curves were obtained for the other options, Figure 4 , which are discussed later in the paper.

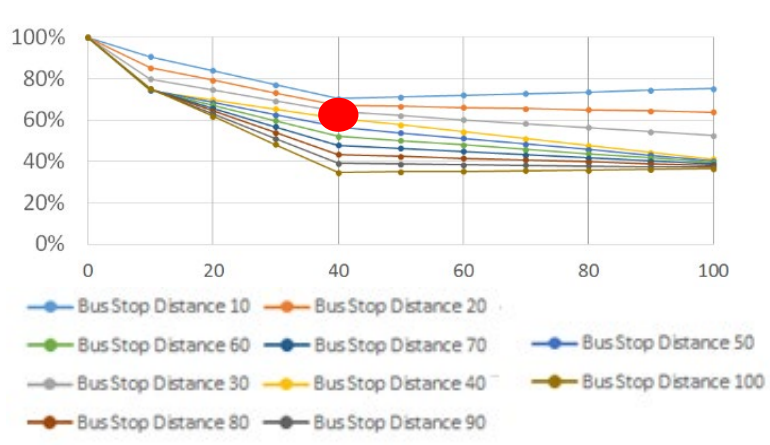

Figure 1 Distribution of Probabilities to use the Zebra crossing (Option 1) in a two way road with busy traffic and rush.

\subsection{Modelling Crossing Behaviour}

Agents in EXODUS are modelled using a fine network of nodes therefore, the pavement area around the road network is discretised in this fashion. In EXODUS the nodes are linked by a system of arcs. The road network area is represented by a network of links and vertices, as in OpenStreepMap [25], with polygonal areas representing the area of each road segment and separate polygons for junctions [21]. A road segment is defined as any straight section of the road network.

While pedestrian agents move using the fine nodal model on the pavement area, on the road, they move using a continuous spatial movement algorithm utilising the hybrid spatial capabilities in EXODUS [26]. A continuous spatial movement algorithm was selected for the road area as it consists of a well-defined region and provides greater flexibility for crossing at arbitrary angles. Since the agents need to be aware of approaching vehicles and other pedestrian agents on the road, a traffic lane based collision avoidance algorithm tied to the road network is utilised. In contrast, the fine nodal model is optimised more for checking for collisions (conflicts algorithm [7]) within a locality of adjacent nodes, which is around 0.5 to

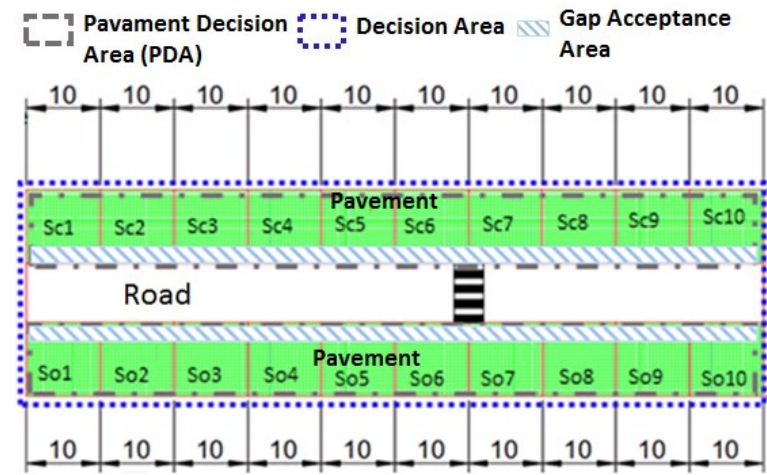

Figure 2 Pavement Area Allocation Types 0.7 metres. Furthermore, a typical vehicle can travel the distance of several fine nodes during a single EXODUS time step when travelling more than $6 \mathrm{~m} / \mathrm{s}(21.6 \mathrm{~km} / \mathrm{h})$, which prevents the utilisation of the nodal spatial representation for the road network without an increase to the EXODUS clock rate. This would in turn require recalibration to the fine node conflict resolution algorithm [7].

When the simulation is initialised, the Gap Acceptance and Pavement Decision Areas, see Figure 2, are automatically identified. The Gap Acceptance Area consists of any nodes directly adjacent to the road and is defined as the region on the pavement where agents can choose to initiate crossing the road, i.e. identify if there is a sufficient gap in the traffic to be able to start to cross the road. The Pavement 
Decision Area (PDA) is a user definable area on either side of the road (divided in Origin and Destination cells $10 \mathrm{~m}$ wide by default, Figure 3 ) where agents consider the possibility of crossing the road.

During the simulation whenever an agent enters, changes their objective or starts responding in a PDA, the model's "Where To Cross" algorithm is utilised. Firstly, it determines whether the agent's projected path will require them to cross the road. If the agent needs to cross the road to achieve their objective, the behaviour algorithm will then identify the agent's Origin and Destination pavement cells and nodes, depicted in Figure 3. The Destination Cell is the cell on the opposite side of the pavement, to the agent, which is nearest to the agent's final objective (Target Destination), e.g. an assembly area or exit.

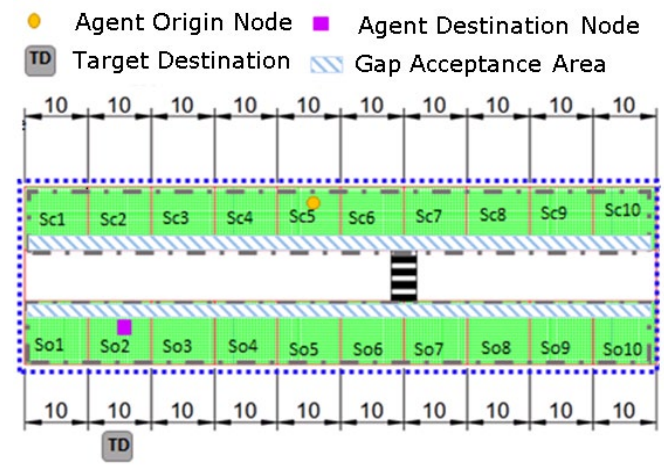

Figure 3 Selection of Target Destination

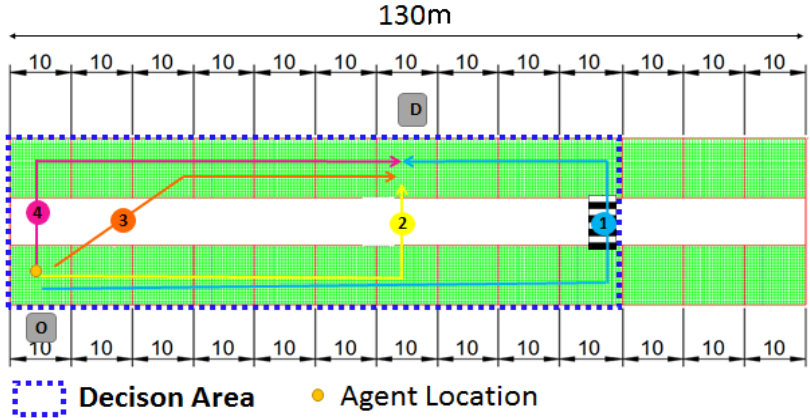

Figure 4 Possible Crossing Options from Agent's Current Origin Cell $O$ to $D$ their Destination.

The Where To Cross Algorithm calculates the agent's Decision Area, default length 100m towards the crossing, and assesses the likelihood for each possible route (Figure 4), being selected. The route selected is based on traffic levels and crossing type, if present, and whether the pedestrian agent is assessed as having urgency (rush, no-rush) and their origin and destination location. The probability of selecting each route, Figure 4, is calculated by linear interpolation [27] using the diagram as shown in Figure 1. The urgency parameter (rush, no rush) is a user define variable.

\subsection{Gap Acceptance (When to Cross)}

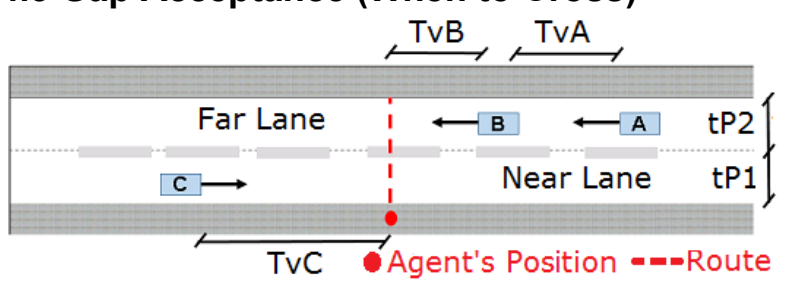

Figure 5 Gap Acceptance Parameters (two way)

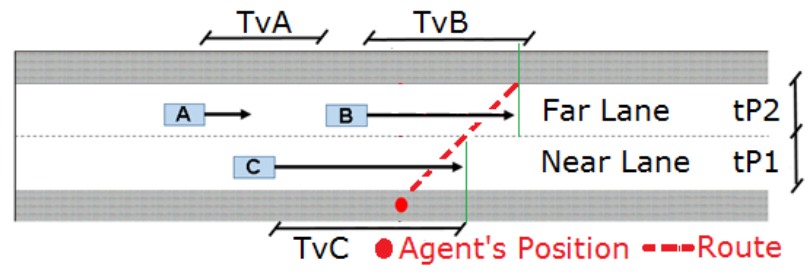

Figure 6 Diagonal Gap Acceptance Model (two way)

When a pedestrian agent enters the Gap Acceptance Area of the pavement and has chosen to Cross the Road (e.g. Options 3 and 4, Figure 4) they will assess the possibility of crossing the road, utilising a Gap Acceptance Model. The Gap Acceptance Model allows the agent to choose to cross all lanes, when safe to do so, in one go, known as double gap [11] or one stage crossing [13]. Alternatively, they may choose to cross lane by lane, known as rolling gap [10] or risk-taker [11], which is selected if the agent is consider as a risk-taker. The percentage of the population which is considered as a risk-taker is a user definable value. How to categorise risk-takers for mixed populations of different age and ability is left for further research.

When an agent looks to cross the road they consider vehicles in the near and far lane, only the nearest vehicle in the near lane and the first two vehicle in the far lane are considered. The key parameters are shown in Figure 5, for perpendicular crossing, and diagonal crossing in Figure 6. The $T v A$, 
parameter, time gap in seconds between vehicles $\mathrm{A}$ and $\mathrm{B}$ in the far lane plus $T v B$ and $T v C$ the time in seconds for vehicles $\mathrm{C}$ and $\mathrm{B}$ to reach the agent's location (first gap near and far).

The pedestrians will use the Double Gap Model if they are identified as risk-averse; hence, will try to cross only when safe to cross both lanes in one go. This is when both the times to cross the first lane $(t P l)$ and the second lane $(t P 2)$ are less than the vehicle gap in the near lane $(T v C)$ and in the far lane $(T v B)$. If this condition is met a probability is calculated to accepting this gap.

The Rolling Gap Model is used when the agent is considered a risk-taker. In this case the time to cross the first lane $(t P l)$ is less than the near gap $(T v C)$ but the time to cross the second lane is greater than the first gap far $(T v B)$ the agent will look at the second gap far $(T v A)$. If this condition is met a probability is calculated to accepting the near gap. If the gap is accepted, the agent will cross the first lane, then when they reach the middle of the lanes, the gaps in the far lane is reassessed. Hence the behaviour modelled is where the agent will cross the first lane and then optionally wait in the middle before continuing if in the second instance the far gap is not accepted.

If a pedestrian agent fails to cross within the time specified by their patience attribute (1-30 seconds) [7] they will reassess Where to Cross and may choose to move further along the pavement and attempt to cross at a different location. When a pedestrian agent reaches a pavement Origin/Destination cell where a Pedestrian Crossing is located, and have chosen to use the crossing, they will move and wait at a location as near as possible the space in front of the crossing, i.e. the nearest EXODUS free fine node location adjacent to the pedestrian crossing.

In the model the Crossing acts as an agent which either allows the pedestrian or vehicles to pass, the decision to cross is not left to the individual agents. In the model currently two types of crossings have been implemented Zebra (Pedestrian Controlled) and a Signalised one.

\section{Demonstration Case}

These behaviours are demonstrated on a large transport hub, namely a train station, where the occupants have been instructed to head towards a place of safety away from the building. Since the focus of the demonstration case is on the crossing behaviours, only a section of the station will be considered, where the occupants evacuate through a main exit into the street. Once outside, they will have to cross a busy road to reach the designated assembly area.

There are three scenarios, which are examined, two cases where there is a road between the station exit and the assembly area that the people will need to cross. For comparison a base case is also given, where the road is removed and people stay on a paved surface to reach the assembly area, Scenario 1. The population in all three scenarios is 2098 with a response time of 0 to 30 seconds. In Table 1 a summary of the key model parameters are summarised.

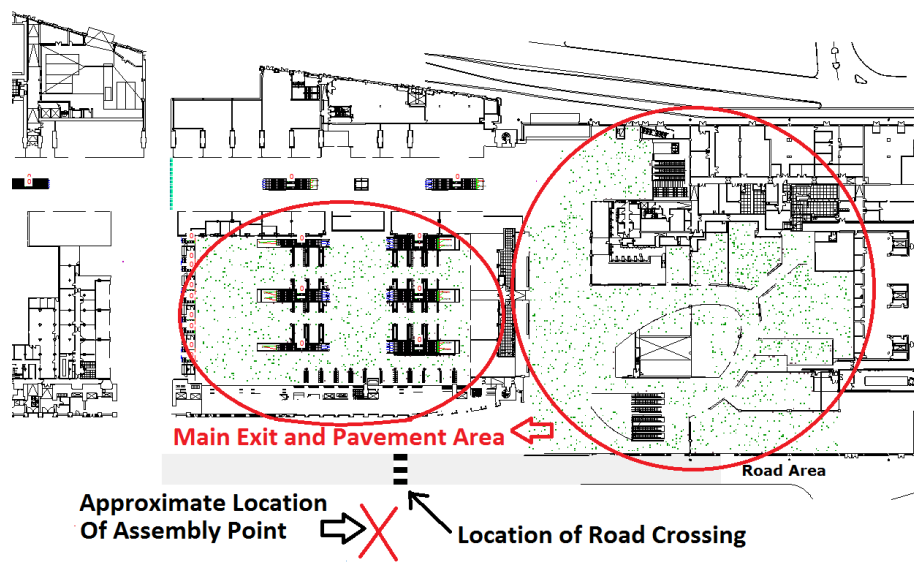

Figure 7 Station Area being evacuated. The Red Circles indicate where the people assigned to use the Main Exit and Assembly

Point are initially located within the station.

Table 1 Simulation Characteristics

\begin{tabular}{|c|c|c|c|}
\hline Scenario & Road & Crossing & Distances \\
\hline 1 & No & None & $\begin{array}{l}\text { Exit to Assembly } \\
\text { Location } 61 \mathrm{~m}\end{array}$ \\
\hline 2 & \multirow{2}{*}{$\begin{array}{c}\text { Yes } \\
2 \text { Lanes (8.5m wide) } \\
\text { Traffic Busy[29] }\end{array}$} & Zebra & \multirow[b]{2}{*}{$\begin{array}{l}\text { Exit to Crossing } 35.5 \mathrm{~m} \text {. } \\
\text { Crossing to Assembly }\end{array}$} \\
\hline 3 & & $\begin{array}{c}\text { Signalised } \\
\text { (Red } 15 \mathrm{~s}, \text { Red Amber }\end{array}$ & \\
\hline
\end{tabular}




\begin{tabular}{|l|l|l|l|}
\hline & Risk Takers 50\%, in rush (100\%) & $\begin{array}{c}3.5 \mathrm{~s}, \text { Green 30s, } \\
\text { Amber 3.5s) [28] }\end{array}$ & Location 29.5m \\
\hline
\end{tabular}

In each of the two road crossing scenarios different pedestrian crossings types will be considered, an un-signalised Zebra Crossing, Scenario 2, and a signalised pedestrian type crossing, Scenario 3. Figure 7 shows the geometry of the station area being evacuated, the assembly location together with road area being modelled and crossing location.

\subsection{Results Analysis and Limitations}

Each of the three scenarios was run a total of 50 times, which was sufficient to get $95 \%$ Confidence Interval on the mean simulation time at a tolerance of 5\%, Figure 8 shows the base case Scenario 1 after 90 seconds, where agents are starting to assemble outside the station. Figure 9 depicts Scenario 3 after $120 \mathrm{~s}$.

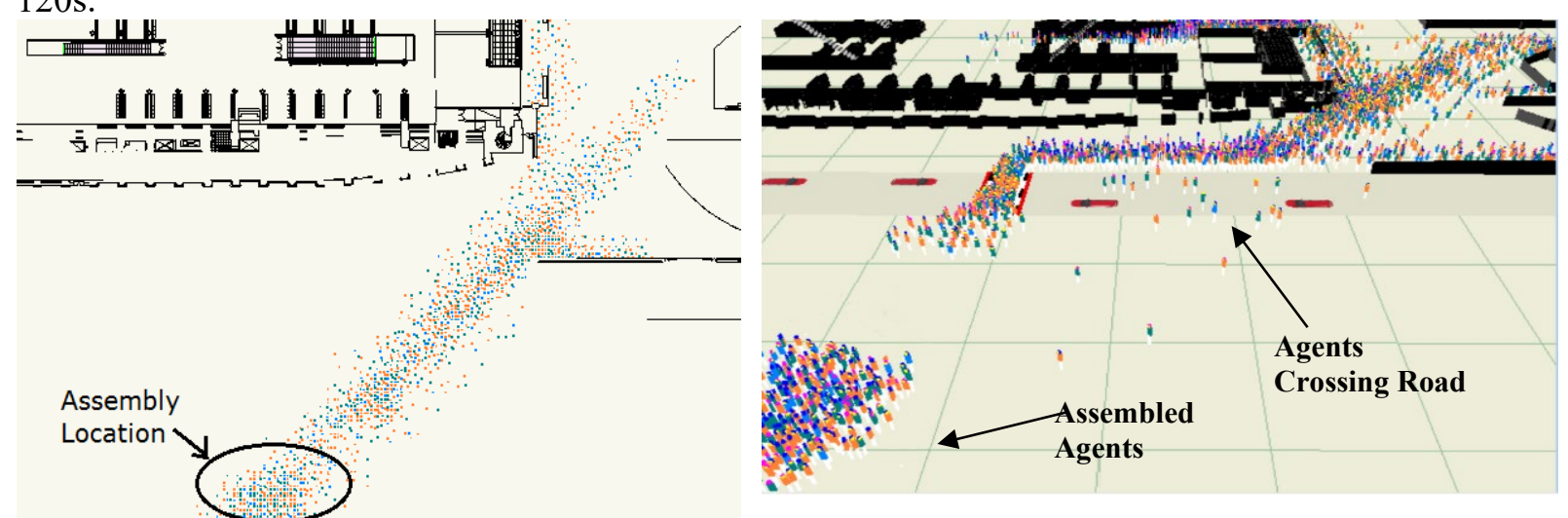

Figure 8 Scenario 1 - Assembly of agents when no road is present (90 seconds into simulation)

Figure 9 Scenario 3 - Agents Crossing at Signalised crossing (120 Seconds). Signals showing red to traffic.

The summary results of the three scenarios are shown in Table 2. The main observation here is that scenarios 1 and 2 mean assembly times are relatively close when compared to Scenario 3, the Signalised Crossing. This is further confirmed when looking at the evacuation curves of scenarios 1 and 2, Figure 10, which show a similar pattern.

\begin{tabular}{|c|c|c|c|}
\hline \multicolumn{2}{|c|}{ Table 2 Simulation Results } \\
Scenario & $\begin{array}{c}\text { Assembly Time (s) } \\
\text { Mean } \pm \text { SD } \mathbf{( 9 5} \% \mathbf{C I})\end{array}$ & $\begin{array}{c}\text { Distance Travelled (m) } \\
\text { Mean [Range] }\end{array}$ & $\begin{array}{c}\text { Percentage Using } \\
\text { Crossing } \\
\text { Mean [Range] }\end{array}$ \\
\hline Scenario 1 & $239.4 \pm 3.1(238.6$ to 240.3$)$ & $138.62[47.05,234.20]$ & N/A \\
\hline Scenario 2 & $280.1 \pm 4.1(278.9$ to 281.2$)$ & $161.65[59.84,243.10]$ & $91.29 \%[90.3,92.5]$ \\
\hline Scenario 3 & $625.9 \pm 16.6(621.4$ to 630.6$)$ & $173.59[59.71,263.30]$ & $95.20 \%[94.27,96.4]$ \\
\hline
\end{tabular}

The reason why the evacuation times and profile from Scenario 2, Zebra Crossing, is closer to, Scenario 1, is that once the agents start to use the Zebra Crossing, the traffic flow is effectively blocked. This allows the agents to proceed continuously across the road, until everyone from the station has evacuated and crossed the road, see the profile of Zebra Crossing usage Figure 10. Only once everyone has crossed the road can the traffic then continue. This means the impact of the road delaying people reaching the assembly areas is only impacted by the additional travel distance required to use the Zebra Crossing to reach the assembly area, see Table 2.

Looking at the crossing usage profile for the Signalised Crossing, see Figure 10 it can be seen that there is a stepped profile, unlike the Zebra Crossing, Figure 10. This means the time for the agents to cross the road is interrupted at intervals of approximately 22 seconds (i.e. time to go from green to red, then back to green). Once the traffic signal has completed its red cycle the agents have around 30 seconds to cross the road. This cycle of stop and go, has an effect on the agents' movement, which increases 
congestion on the pavement area, as can be seen when comparing Figure 8, where there is no crossing, to Figure 9, with the signalised crossing.

Furthermore, it is evident that a higher than expected proportion of the pedestrians who use the crossing (around 90\%), in both the Signalised and Zebra Crossing cases. This percentage is greater than the one shown in Figure 1. This is most likely due to the position of the crossing being located towards the assembly area. Furthermore, when the pavement is congested, agents have difficulty moving towards the edge of the pavement to cross the road. This means they re-evaluate the probability of where to cross, hence increasing the likelihood of the agents moving towards the pedestrian crossing.

The model has not been fully calibrated, only basic functional testing and verification has been performed so

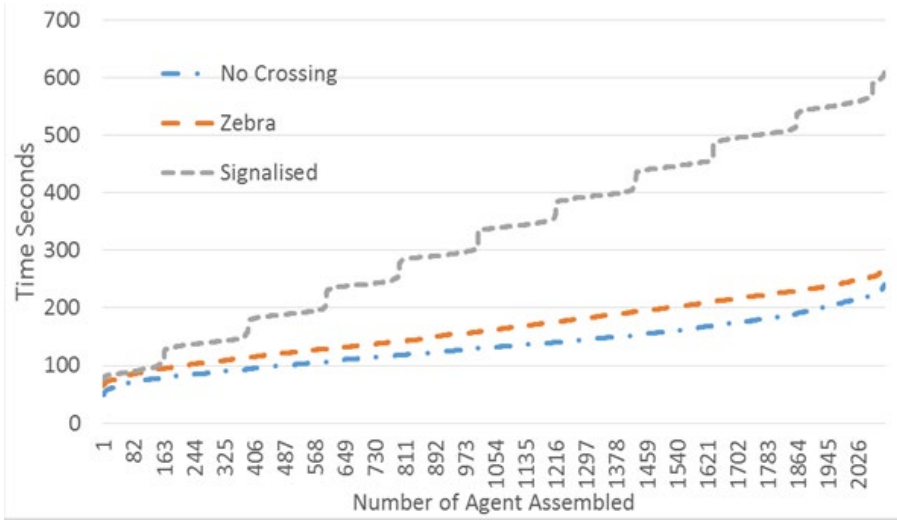

Figure 10 Scenario Evacuation Curves - Time to Assemble far. Since no model validation has yet been carried out, the results can only be considered as indicative. The probability of crossing the road is mainly based on the pedestrian agent's distance from the pedestrian crossing, traffic and time pressure and does not consider the crowd density on the pavement. In crowded situations people are probably more likely to cross outside the pedestrian crossing to escape congestion. So further research could be focused on whether the crowd density could also be factored into the agent's crossing decision, this would likely have an impact on the results presented in the demo case presented here.

Further work will be looking at the above limitations as well as examining the impact on the model of varying the likelihood of crossing outside the pedestrian crossing. This will be done in addition to investigating how to connect the crossing and evacuation model to a third-party vehicle model, for example SUMO [30], which is one such model under consideration.

\section{Conclusions and Final Remarks}

This research has incorporated a number of key model developments and behaviours that are required to simulate the interactions of pedestrians with vehicles after exiting from a building during an emergency, which represents a unique effort to bridge the evident behavioural gaps identified in the integrations of emergency pedestrian models with traffic simulations. This work allows agents to decide when and where to cross a road once they have evacuated from a building during an emergency, incorporating psychological factors that affect their decision process. Further work is required for calibration and to examine the sensitivity of the various model crossing parameters, before moving onto model validation.

\section{Acknowledgements}

Part of this research was funded under a University of Greenwich VC Scholarship.

\section{References}

[1] H.J. Hurley et al. (Ed.), SFPE Handbook of Fire Protection Engineering, Springer, New York, 5th edition, ISBN: 978-1-4939-2564-3, 2016.

[2] London Fire Brigade, (April 2015) Holborn fire shows how complex London can be says capital's fire chief [Online] Available: http://www.london-fire.gov.uk/news/LatestNewsReleases holbornfireshowshowcomplex.asp, [Accessed: 7/01/2018].

[3] HSG154, Managing crowds safely, A guide for organisers at events and venues, (Second edition), ISBN 978071761834 7, 2000. 
[4] L.D. Han, F. Yuan, "Evacuation modeling and operations using dynamic traffic assignment and most desirable destination approaches", in Proceedings of the 84th Annual Meeting Transportation Research Board, Washington, DC, USA, November 2005.

[5] G. Lämmel, H. Klüpfel, K. Nagel, "The MATSim Network Flow Model for Traffic Simulation Adapted to Large-Scale Emergency Egress and an Application to the Evacuation of the Indonesian City of Padang in Case of a Tsunami Warning", Chapter 11, pages 245-265, Emerald Group Publishing Limited, DOI:10.1108/9781848557512-011, 2009.

[6] LEGION, (2017) Pedestrian and Traffic Simulation Integrated Into a Single Software Application [Online], Availiable: (http://www.legion.com/pedestrian-traffic-simulation-integrated -into-a-single-software-application, [Accessed: 3 Jan 2018].

[7] E Galea, M Owen, P Lawrence, “The EXODUS Model”, Fire Engineers Journal, pp.26-30, July 1996.

[8] E.R. Galea, P.J. Lawrence, L. Fillippidis, D. Blackshields, D. Cooney, "buildingEXODUS V 6.1 Theory Manual", Fire Safety Engineering Group, University of Greenwich, 2012.

[9] T. Wang, Studyof Pedestrian - Vehicle Interaction Behaviour by Microscopic Simulation Modelling, Doctoral Thesis, University of Southampton, Faculty of Engineering and the Environment, 2012.

[10] M. Brewer, K. Fitzpatrick, J. Whitacre, D. Lord, D, "Exploration of Pedestrian Gap-Acceptance Behavior at Selected Locations", Transportation Research Record, 1982(1), 132-140. doi:10.3141/1982-18, 2006.

[11] L. Song, M.C. Dunne and J.A. Black, "Models of Delay and Accident Risk to Pedestrians,Transportation and Traffic Theory", Proceedings of the Twelfth International Symposium,Elsevier Science, 2003.

[12] D. Sun, S. Ukkusuri, R. Benekohal, S. Waller, "Modeling of motorist-pedestrian interaction at uncontrolled mid-block crosswalks", Proceedings of $82^{\text {nd }}$ Annual Meeting of the Transportation Research Board, Urbana, November 2002.

[13] M. Paul and P. Rajbonshi, "A Comprehensive Review on Pedestrian Gap Acceptance at Unsignalized Road" International Journal of Engineering Research \& Technology (IJERT), Vol. 3 Issue 11, November-2014, ISSN: 2278-0181, November 2014.

[14] E. Ronchi, S. Gwynne, G. Rein, R. Wadhwani, P. Intini and A. Bergstedt, "e-Sanctuary: Open Multi-Physics Framework for Modelling Wildfire Urban Evacuation", Fire Protection Research Foundation Report, FPRF-2017-22, December 2017.

[15] P. Alvarez, V Alonso, "Using microsimulation software to model large-scale evacuation scenarios. The case of Sangüesa and the Yesa dam collapse", Safety Science, Vol. 106 Pages 10-27, https://doi.org/10.1016/j.ssci.2018.02.021,2018.

[16] B.J Schroeder, "A Behavior-Based Methodology for Evaluating Pedestrian-Vehicle Interaction at Crosswalks", Doctoral Thesis North Carolina State University, Departement of Civil Engineering, 2008.

[17] V. Pellacini, (2016), "Pedestrian Behaviour Survey", Fire Safety Engineering Group, University of Greenwich [Online] Avialable:https://fseg.gre.ac.uk/surveys, [Accessed: 2 Feb 2018].

[18] A. Dommes, V. Cavallo J. Dubuisson, I. Tournier, F. Vienn, "Cossing a two-way street: comparison of young and old pedestrians", Journal of Safety Research, vol. 50, pp27-34,2014.

[19] X. Zhuang, C Wu, "Pedestrians' crossing behaviors and safety at unmarked roadway in China", Accident Analysis \& Prevention, vol. 43, pp. 1927-1936, 2011.

[20] M. McDonald, J. Wu and M. Brackstone, "Development of a Fuzzy Logic Based Microscopic Motorway Simulation Model", in Proceedings of the IEEE Conference on Intelligent Transportation Systems (ITSC97), Pages 82-87 Boston, USA, DOI: 10.1109/ITSC.1997.660454, 1997.

[21] P.J. Lawrence, L. Flippidis, A. Veeraswamy, E. Galea E, "Utilising OpenStreetMap for Urban Evacuation Analysis", in Proceedings of the 24th GIS Research UK (GISRUK 2016) conference, University of Greenwich, March 2016.

[22] G. Yannis, N. Technical, "Pedestrian gap acceptance for mid-block street crossing", in Proceedings of the 12th World Conference for Transportation Research, pages 1-11, 2010.

[23] R. Fahy, G. Proulx, "Panic and Human Baheviour in Fire", Proceedings of the 4th International Symposium on Human Behaviour in Fire Cambridge, UK: Robinson College, 2009.

[24] X. Chu, M. Guttenplan. M Baltes, "Why People Cross Where They Do" Transportation Research Record: Journal of the Transportation Research Board, 160(03), Januray 2004.

[25] OpenStreetMap (2018) OpenStreetMaps [online], Avialable: https://www.openstreetmap.org, [Accessed: 27 April $n$ 2018].

[26] N. Chooramun, P. Lawrence, E. Galea E, "Evacuation simulation using Hybrid Space Discretisation and Application to Large Underground Rail Tunnel Station", Physical Sciences Reviews, Vol. 2, Issue 7, DOI:10.1515/psr-2017-0001, Aug 2017.

[27] V. Airault, S. Espie, "Behavioural model of the pedestrian interaction with road traffic", Association of European Transport and Contributors, 2005.

[28] R. Walker,M. Winnett, A. Martin, J. Kennedy, "Puffin Crossing Operation and Behaviour Study", Transport Research Laboratory project report for the London Road Safety Unit, Transport for London, 2005. 
[29] J. Hine, "Assessing the impact of traffic on behaviour and perceptions of safety using an in-depth interview technique", Journal of Transport Geography, 4(3), 179-197, 1996.

[30] D. Krajzewicz, J. Erdmann, M. Behrisch, and L. Bieker, "Recent Development and Applications of SUMO - Simulation of Urban Mobility" International Journal On Advances in Systems and Measurements, 5 (3\&4):128-138, December 2012. 